\title{
Astrometric and Photometric Variability in Quasars
}

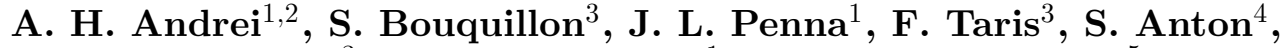 \\ J. Souchay $^{3}$, J. I. B. Camargo ${ }^{1}$, D. N. da Silva Neto ${ }^{5}$, \\ R. Vieira Martins ${ }^{1}$, M. Assafin ${ }^{2}$, and S. dos Reis Carvalho Pinto ${ }^{1,6}$ \\ ${ }^{1}$ Observatório Nacional/MCT, R. Gal. Jose Cristino 77, Rio de Janeiro, Brazil \\ Email: oat1@on.br \\ ${ }^{2}$ Observatório do Valongo, UFRJ, Brazil \\ ${ }^{3}$ SYRTE/Observatoire de Paris, France \\ ${ }^{4}$ Centro de Investigação em Ciencias Geo-Espaciais/FCUP, Portugal \\ ${ }^{5}$ Universidade Estadual da Zona Oeste-BR, ${ }^{6}$ Universidade Gama Filho, Brazil
}

Keywords. (galaxies:) quasars: general, astrometry, techniques: photometric, reference systems

Quasars are the choicest objects to define a quasi-inertial reference frame. At the same time, they are active galactic nuclei powered by a massive black hole. As the astrometric precision of ground-based optical observations approaches the limit set by the forthcoming GAIA mission, astrometric stability can be investigated. Though the optical emission from the core region usually exceeds the other components by a factor of a hundred, the variability of those components must surely imply some measure of variability of the astrometric baricenter. Whether this is confirmed or not, it puts important constraints on the relationship of the quasar's central engine to the surrounding distribution of matter. To investigate the correlation between long-term optical variability and what is dubbed as the "random walk" of the astrometric center, a program is being pursued at the WFI/ESO $2.2 \mathrm{~m}$. The sample was selected from quasars known to undergo large-amplitude and long-term optical variations (Smith et al. 1993; Teerikorpi 2000). The observations are typically made every two months. The treatment is differential, comparing the quasar position and brightness against a sample of selected stars for which the average relative distances and magnitudes remain constant. The provisional results for four objects bring strong support to the hypothesis of a relationship between astrometric and photometric variability. A full account is provided by Andrei et al. (2009).

\section{Acknowledgements}

A.H.A. thanks CNPq grant PQ-307126/2006-0. M.A. thanks grant CNPq 478318/2007-3. J.I.B.C. thanks grant E-26/100.229/2008/FAPERJ.

\section{References}

Andrei, A. H., Bouquillon, S., Camargo, J. I. B., Penna, J. L., Taris, F., Souchay, J., da Silva Neto, D. N., Vieira Martins, R., \& Assafin, M. 2009, in Proceedings of the Journees 2008 Systemes de reference spatio-temporels, ed. M. Soffel and N. Capitaine, (Paris: LohrmannObservatorium and Observatoire de Paris), p. 199

Smith, A. G., Nair, A. D., Leacock, R. J., \& Clements, S. D. 1993, AJ, 105, 2, 437

Teerikorpi, P. 2000, A\&A A, 353, 77 